\title{
A Pigment Analysis Tool for Hyperspectral Images of Cultural Heritage Artifacts
}

\author{
Di Bai ${ }^{\mathrm{a}}$, David W. Messinger ${ }^{\mathrm{a}}$, and David Howell ${ }^{\mathrm{b}}$ \\ ${ }^{a}$ Chester F. Carlson Center for Imaging Science, Rochester Institute of Technology, Rochester, \\ NY, USA \\ ${ }^{\mathrm{b}}$ Bodleian Library, University of Oxford, Broad St, Oxford OX1 3BG, UK
}

\begin{abstract}
The Gough Map, in the collection at the Bodleian Library, Oxford University, is one of the earliest surviving maps of Britain. Previous research deemed that it was likely created over the 15th century and afterwards it was extensively revised more than once. In 2015, the Gough Map was imaged using a hyperspectral imaging system at the Bodleian Library. The collection of the hyperspectral image (HSI) data was aimed at faded text enhancement for reading and pigment analysis for the material diversity of its composition and potentially the timeline of its creation. In this research, we introduce several methods to analyze the green pigments in the Gough Map, especially the number and spatial distribution of distinct green pigments. One approach, called the Gram Matrix, has been used to estimate the material diversity in a scene (i.e., endmember selection and dimensionality estimation). Here, we use the Gram Matrix technique to study the within-material differences of pigments in the Gough map with common visual color. We develop a pigment analysis tool that extracts visually common pixels, green pigments in this case, from the Gough Map and estimates its material diversity. It reveals that the Gough Map consists of at least six kinds of dominant green pigments. Both historical geographers and cartographic historians will benefit from this work to analyze the pigment diversity using HSI of cultural heritage artifacts.
\end{abstract}

Keywords: hyperspectral imagery; pigment analysis; dimensionality estimation

\section{INTRODUCTION}

The Gough Map was created in the 15 th century. ${ }^{1-4}$ It is one of the earliest surviving maps of Britain in geographically recognizable form. The substrate of the map is a combination of lamb skin and sheepskin and in this map, there are more than 600 towns indicated in red signs, a depiction of waterways indicated in green, and distances between the towns. ${ }^{1-3}$ Previous research revealed that this map was revised after it was created. ${ }^{1,3}$ According to Delano-Smith, et al (2017), the map was created in three different layers during three different periods. After its creation, there are two revision steps. Even though the map appears to have been revised over approximately 100 years, evidence of similar signs and symbols shows that in each step similar pigments and methods were utilized to create and update the map. ${ }^{3}$

Questions about the map arise such as faded text enhancement for reading and pigment analysis for estimation of material diversity in the Gough Map. In an effort to answer such questions, hyperspectral images (HSI) of the map were collected at the Bodleian Library, Oxford University in 2015. The collection was done in six "chips" to cover the entire map with sufficient spatial resolution. Each chip has 334 spectral bands over the Vis-NIR spectrum ranging from 400nm to $1000 \mathrm{~nm}$. The size of each chip is 1600 (pixels) x 5512 (pixels). More collection details will be introduced in Section 3.1. The analysis of the hyperspectral imagery contributes to the codicological studies of the map (i.e., understanding the tools, techniques, and timeline of its creation). This paper presents an approach to estimating the material diversity of one of the primary pigments used on the map, the green inks.

Further author information: (Send correspondence to Di Bai)

Di Bai: E-mail: db3641@rit.edu, Telephone: 15857543879

Algorithms and Technologies for Multispectral, Hyperspectral, and Ultraspectral Imagery XXIII

edited by Miguel Velez-Reyes, David W. Messinger, Proc. of SPIE Vol. 10198, 101981A

(c) 2017 SPIE $\cdot$ CCC code: 0277-786X/17/\$18 - doi: 10.1117/12.2261852

Proc. of SPIE Vol. 10198 101981A-1 
For faded text analysis in cultural heritage, previous research has used multispectral reflectance, fluoresence, and transmission imaging ${ }^{5-12}$ to enhance faded text for reading. For example, Easton and his collaborators used a Megavision Imaging System to collect and generate multispectral images of artifacts and then used several image processing techniques to enhance the images. Methods included unsupervised/supervised classification, principal component analysis (PCA), independent component analysis (ICA), spectral pseudoinverse calculation, deterministic renderings in pseudo-color, and spectral un-mixing to enhance faded texts from historical manuscripts, such as the Herculaneum Papyri and Archimedes Palimpsest. ${ }^{5-12}$ For pigment analysis, Zhao $(2008)^{13}$ developed algorithms for image segmentation and pigment mapping for multispectral images of the artistic paintings, such as "Starry Night". ${ }^{13}$ Her methods consist of a spectral reconstruction algorithm based on Wyszecki's hypothesis and a pigment mapping algorithm based on Kubelka-Munk turbid media theory that can predict spectral reflectance for a specimen from the optical properties of its constituent pigments. The output of her pigment mapping method was a set of spatial distributions of pigments composing the painting. Comelli et al (2007) developed a fluorescence multispectral imaging system to analyze surfaces of Renaissance frescoes. ${ }^{14}$ This multispectral system utilized UV lamps for excitation and a liquid crystal filter attached to a CCD as the detector. Their multispectral data is over the fluorescence spectrum. This system can detect and differentiate binders and pigments with specific fluorescence properties. Previous research on faded text analysis and pigment analysis of cultural heritage artifacts was based on multispectral imaging, but in this research, we analyze the within material diversity of pigments in the HSI of the Gough Map to aid in understanding the techniques and timeline of the creation of the map.

This paper is organized as follows. Section 2 describes the MaxD and Gram Matrix techniques used to estimate material diversity in the HSI of the Gough Map. Section 3 describes application using this technique to estimate the material diversity of green pigments in the Gough Map and presents the results. Section 4 summarizes the results and findings.

\section{MATERIAL DIVERSITY ESTIMATION}

Material diversity estimation of a data set in the spectral domain can be assumed to be related to the dimensionality of the data, a generally reasonable and applicable assumption. The dimensionality of a data set can be defined as the minimum number of free variables required to represent the data without losing information. ${ }^{15}$ Many approaches have been used to estimate the dimensionality of a data set, ${ }^{15-22}$ but the most common in hyperspectral imagery is through the Principal Components Analysis, ${ }^{18,19}$ which equates the dimensionality of the data with the significance of a dimension in the transformed space through the magnitude of its associated eigenvalue. Other methods such as box-counting and point density have also been proposed. ${ }^{23}$

In this paper, we use the approach developed by Messinger, et al (2011) ${ }^{16}$ and applied by Canham, et al $(2011)^{17}$ to estimate the number of green pigments in the HSI of the Gough Map. The Gram Matrix is used in this approach, which is a similarity matrix representation of vector data that has unique properties useful when estimating the dimensionality of high-dimensional data. In this approach, endmembers are obtained from the scene to represent the $n$ "pure" materials in the scene, and $n$ has to be larger than a priori estimate of the number of materials (but smaller than the number of spectral bands of the image). The MaxD algorithm is used to automatically extract the $n$ endmembers from the scene. ${ }^{24}$ The Gram Matrix is an $m \times m$ matrix, where $m$ is the number of vectors in the set. The $(i, j)^{t h}$ element of the Gram matrix is the inner product of the $i$ and $j$ vectors in the set, such that

$$
G_{i, j}=\left\langle x_{i}, x_{j}\right\rangle .
$$

An important property of the Gram Matrix is that the determinant of $G$, known as the Gramian, is the square of the volume of the parallelepiped that encloses the vector set. Consequently, when the Gramian approaches zero, the vectors in the set are no longer linearly independent. For hyperspectral data this indicates the number of distinct materials in the scene. More details on using this approach to estimate the number of distinct materials in a hyperspectral scene can be found in the literature. ${ }^{16,17,20}$

In this paper, we first cut each HSI chip of the Gough Map into top and bottom sub-chips to speed computation and then use a Mahalanobis distance classifier to classify each sub-chip of the Gough Map into three parts, red pigment (towns and roads), green pigment (waterways, coast and ocean) and gray background. After that, 
the green pixels from the Gough Map are extracted and their dimensionality is estimated by the Gram Matrix technique. The research objective is to estimate the number of distinct green pigments utilized in the creation and revision of the entire Gough Map and to ascertain if there is a spatial pattern in the distribution of each pigment type across the map. The Gram matrix technique enables us to estimate within-class dimensionality of the data and separate out potentially different green pigments based on their spectra, despite their common visually "green" appearance.

\section{APPLICATION TO THE GOUGH MAP}

In this paper, our goal is to estimate the within material diversity of the green pigments in the Gough Map through analysis of the hyperspectral imagery. In this section, we introduce the application of the above-described analysis technique to the green pigments.

\subsection{Collection Parameters}

The Gough Map, shown in Figs. 1 \& 2, is drawn on one piece of sheepskin and one lambskin that were originally sewn together, but the stitching is hardly seen. ${ }^{4}$ The actual size of the Gough Map is $55.3 \times 116.4 \mathrm{~cm}$ and the scale is estimated at about 1:1,000,000, or sixteen miles to one inch.

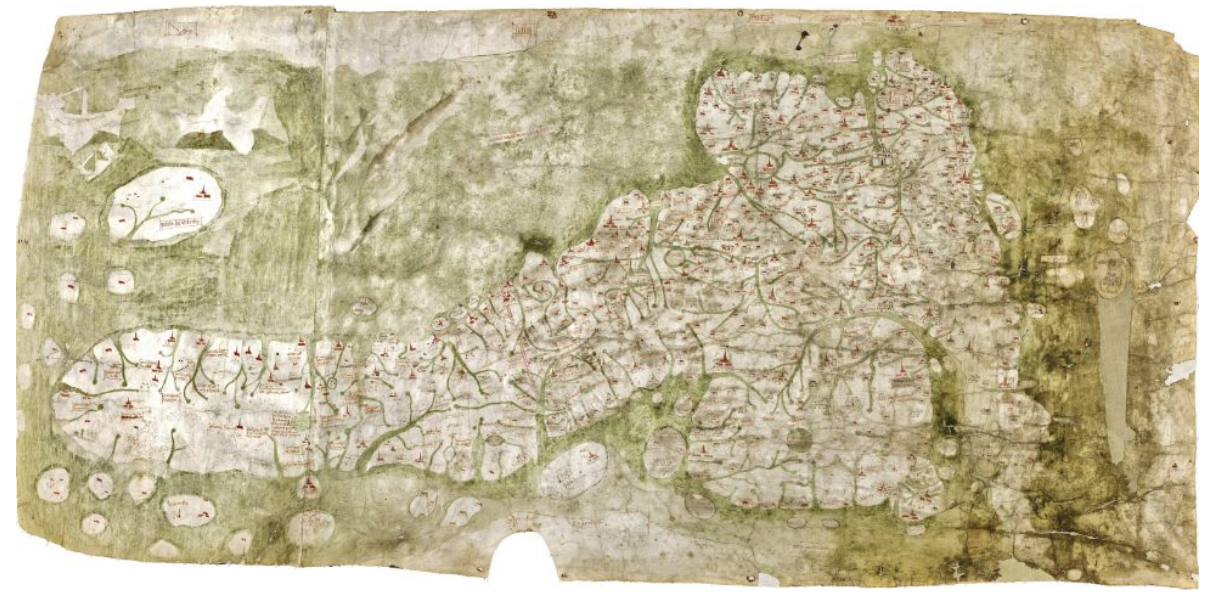

Figure 1. High resolution RGB image of the Gough Map. This map is oriented such that East is at the top of the page and England is to the right. Towns and distances are indicated in red; Ocean, coast and waterways are in green. The substrate of the map is a combination of lamb skin (leftmost quarter of map) and sheepskin (right 3/4 of map). Note the damaged area at the right edge (south of Wales).

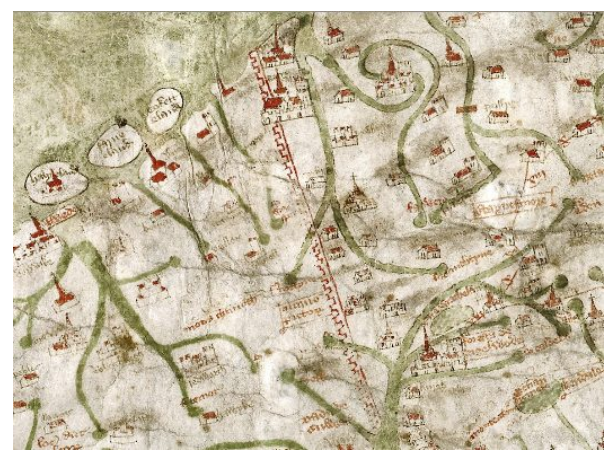

Figure 2. Zoom of the high resolution RGB image of the Gough Map showing in red Hadrian's Wall, town signs, distance indicators, texts and green coast line, waterways. 
To ensure full coverage of the map with sufficient spatial resolution, the Gough Map was imaged in 6 spatially overlapping chips. Fig. 3 shows the RGB images of the six chips of the Gough Map. The overlapping areas are apparent as are the supporting structures around the edges to keep the map relatively flat. For convenience, we

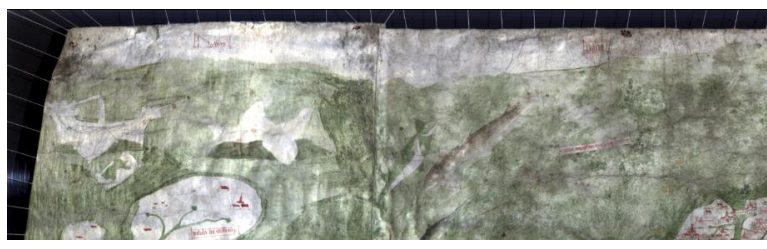

(a)

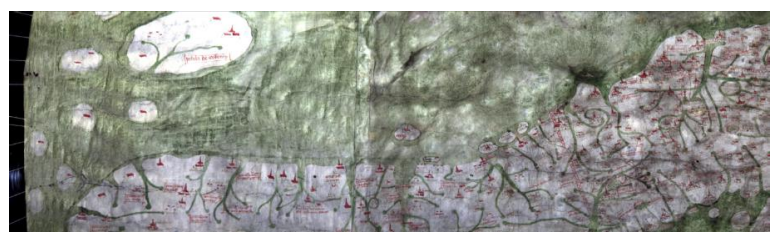

(c)

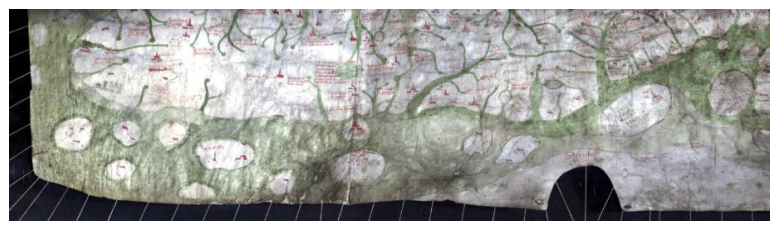

(e)

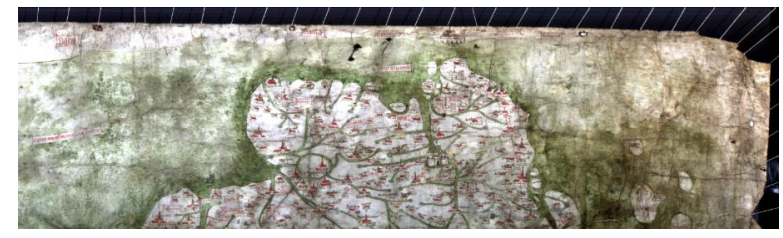

(b)

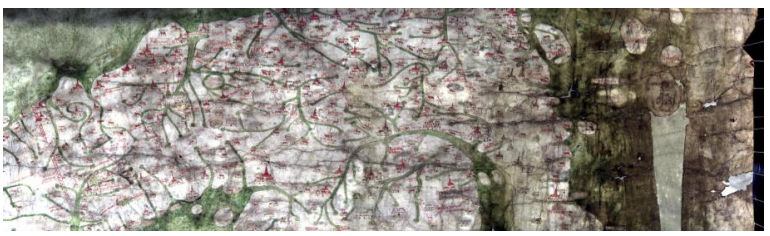

(d)

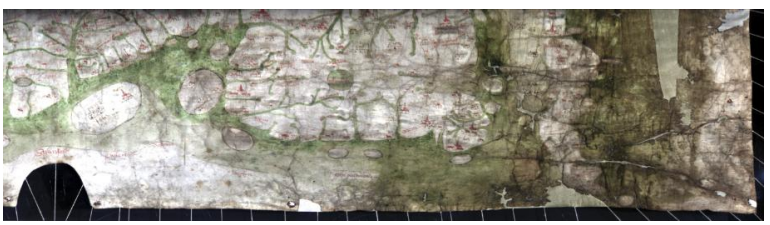

(f)

Figure 3. RGB renderings of the HSI chips representing the collection of the entire Gough Map.

label the six parts as 1L, 1R, 2L, 2R, 3L and 3R as shown in Fig. 4.

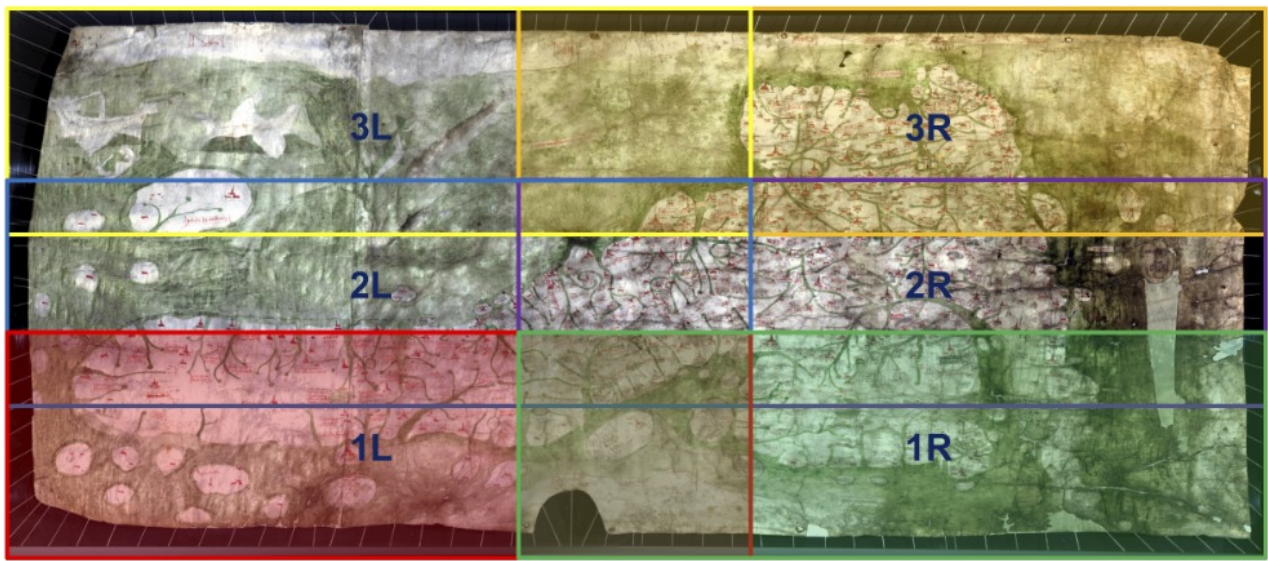

Figure 4. The entire Gough Map with chips labeled as 1L, 1R, 2L, 2R, 3L, 3R.

Each HSI chip has 334 bands and the size of each chip is 1600 (pixels) x 5512 (pixels). The entire Gough Map is about 3743 (pixels) x 8987 (pixels) x 334 (bands). Given that the actual size of the Gough Map is 55.3 $\mathrm{x} 116.4 \mathrm{~cm}$, the spatial resolution in the horizontal direction is approximately $\Delta x \simeq 77$ pixels $/ \mathrm{cm}$, and in the vertical direction is about $\Delta y \simeq 68$ pixels $/ \mathrm{cm}$. In addition, each chip has 334 bands over the Vis-NIR ranging from $398.7 \mathrm{~nm}$ to $1000.3 \mathrm{~nm}$, so that $\Delta \lambda=1.8 \mathrm{~nm}$. 


\subsection{Methodology}

The diversity of the green pigments in the Gough Map is estimated by using the Gram Matrix technique as described above. The methodology of this application is outlined here:

1. Cut each HSI chip of the Gough Map into top and bottom sub-chips, such as sub-chip 1L(top).

2. Sphere the data of each sub-chip by unit magnitude normalizing each pixel to eliminate brightness differences in the reflectance.

3. Use a Mahalanobis distance classifier in ENVI software to extract the green pixels of each sub-chip.

4. Use the MaxD and Gram Matrix techniques to estimate the dimensionality for each sub-chip and compute endmembers.

(a) extract $m$ endmembers $(m \approx 10)$

(b) iterate $i$ from 3 to $m$ endmembers

i. represent the $i$ endmembers in the Gram matrix

ii. compute the convex hull volume for $i$ endmembers by computing the Gramian

iii. save that volume as a function of $i$

(c) the estimated dimensionality of the sub-chip is the number of endmembers where the convex hull volume function approaches zero

5. For each sub-chip, use spectral angle mapper (SAM) to classify the green pixels based on its endmembers and count the number of pixels in each class.

6. Map the classification results back to the image for visual interpretation.

7. Compute global distinct green pigments for the entire Gough Map and re-classify the entire Gough Map.

Details are provided in following subsections on the steps outlined above.

\subsubsection{Cut each HSI chip into sub-chips}

The data size of each HSI chip is about 12GB. Each HSI chip contains 2,700,000 to 5,500,000 green (visually) pixels. To speed computation, we first cut each HSI chip into top and bottom sub-chips. The results are shown in Fig. 5(a-l).

\subsubsection{Sphere data to eliminate brightness differences}

The second step is to sphere the data of each sub-chip of the Gough Map. In each collection, a white reference image for each chip was also captured and consequently, by normalizing out this white reference, the estimated reflectance of each pixel in the Gough Map was obtained. However, there are differences in this process and not all of the chips have the same brightness levels. For example, Fig. 6 shows two images of an overlapping area between chips $3 \mathrm{~L}$ (left) and 2L (right). To compare their spectra, we extract the same green pixel from both $3 \mathrm{~L}$ and 2L (in the red box) and plot their spectra in Fig. 7(a), the result shows that their spectra have significant brightness differences but little color difference. 


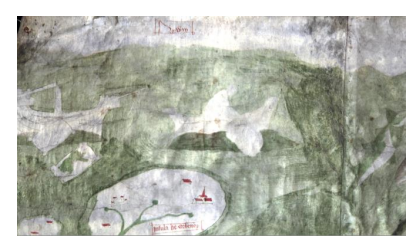

(a)

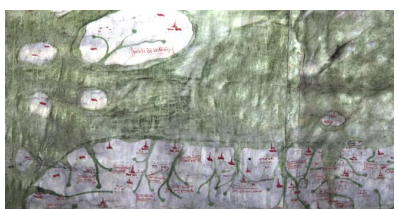

(e)

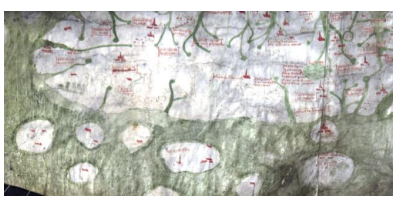

(i)

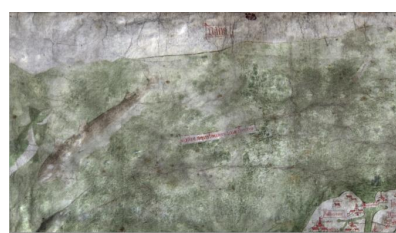

(b)

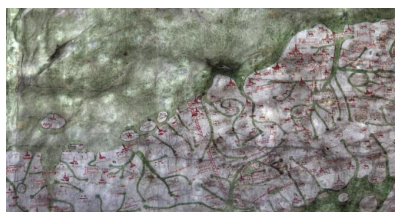

(f)

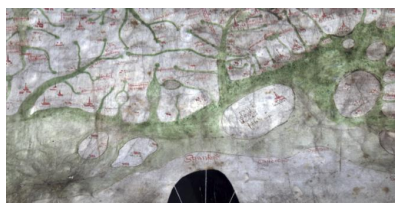

(j)

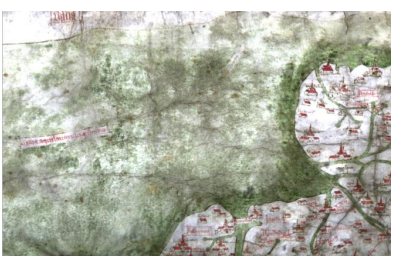

(c)

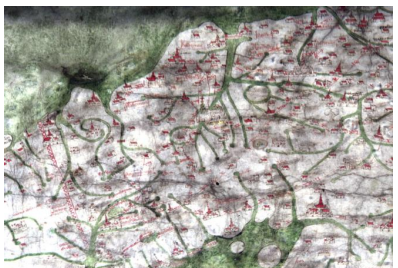

(g)

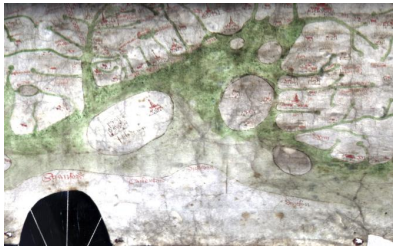

(k)

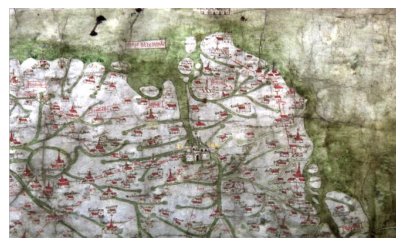

(d)

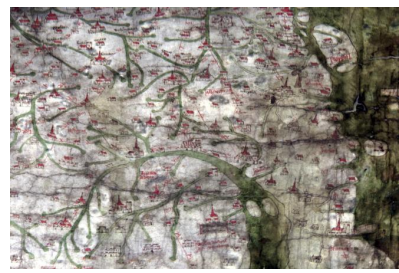

(h)

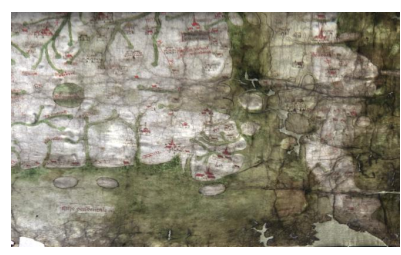

(l)

Figure 5. RGB renderings of the sub-chips of the Gough Map. (a) 3L top (b) 3L bottom (c) 3R top (d) 3R bottom (e) $2 \mathrm{~L}$ top (f) $2 \mathrm{~L}$ bottom (g) $2 \mathrm{R}$ top (h) $2 \mathrm{R}$ bottom (i) $1 \mathrm{~L}$ top (j) $1 \mathrm{~L}$ bottom (k) $1 \mathrm{R}$ top and (l) $1 \mathrm{R}$ bottom.

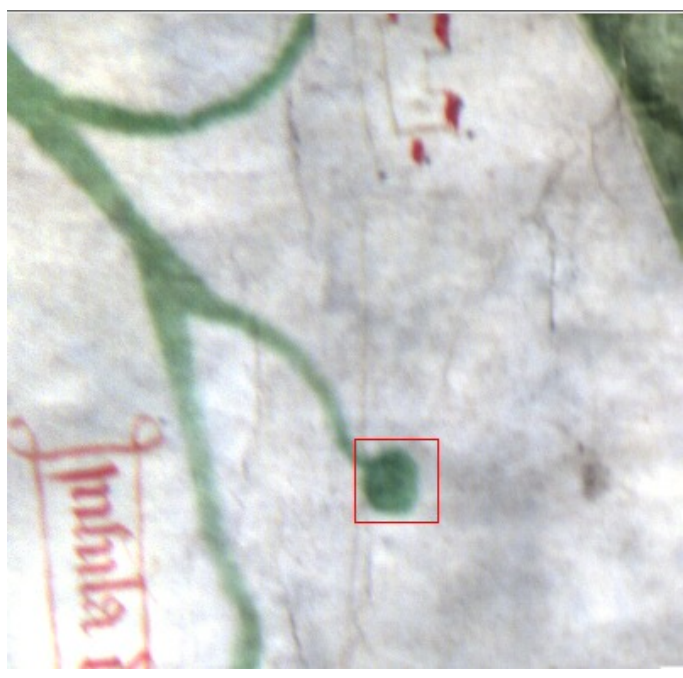

(a)

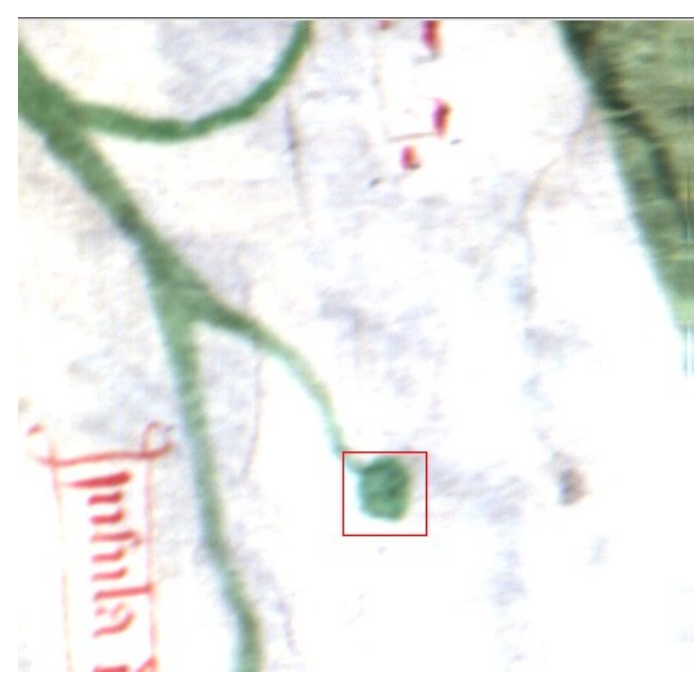

(b)

Figure 6. Example of same area in two different chips of the Gough Map: 3L (a) and 2L (b). Note the brightness differences when same histogram stretch is applied. 
Sphering the data, i.e., unit normalizing each pixel vector, can remove brightness differences in the data set by ensuring each pixel has a vector magnitude of 1 . This results in a normalized reflectance for each pixel. As a comparison, after we sphere the spectra as shown in Fig. 7(b), the brightness differences are removed.

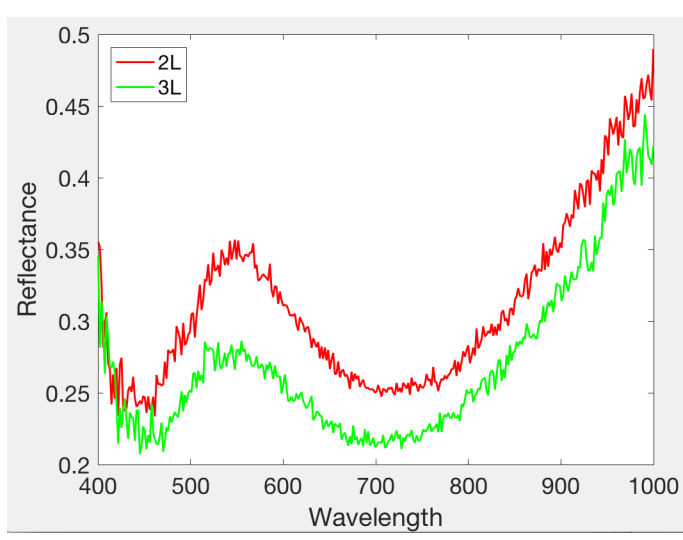

(a)

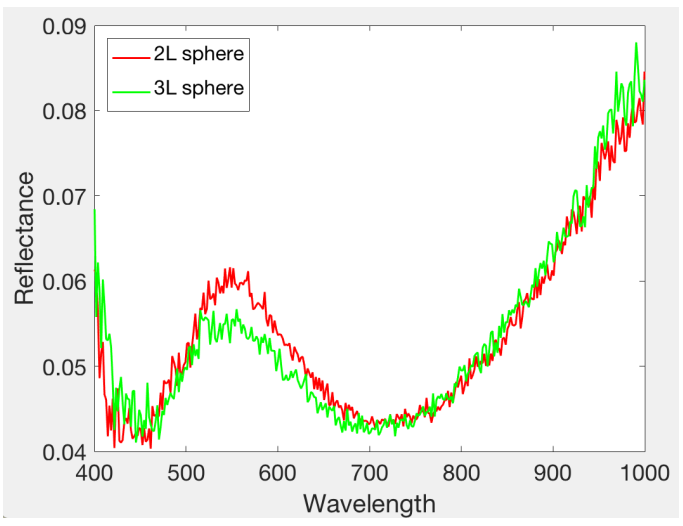

(b)

Figure 7. Spectral differences of common green pixel between 2L and 3L before sphering (a) and after sphering (b).

\subsubsection{Extract green pixels}

The third step is to extract green pixels from the twelve HSI sub-chips of the Gough Map. Here we use a supervised classifier in the ENVI software to classify each sub-chip. For example, in the 2R(top) chip as shown in Fig. 8(a), we first specify three regions of interest (ROI) as shown in Fig. 8(b), the red pigment region, the green pigment region and the white background (the substrate), and then based on the three ROIs, we use a Mahalanobis distance classifier to classify this 2R(top) chip. Fig. 8(c) shows the output of the classification as a gray scale image, which consists of three regions: black (green pigment), white (background), and gray (red pigment). Then we simply add a threshold to convert (c) to a binary image shown in Fig. 8(d), so that the white pixels in (d) represent all the green pixels in the original 2R(top) chip, automatically extracted. Finally, the data of green pixels are exported for endmember analysis and meanwhile, the coordinates of the green pixels are also saved for future mapping in Section 3.2.6.

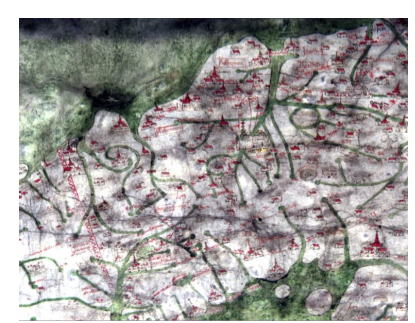

(a)

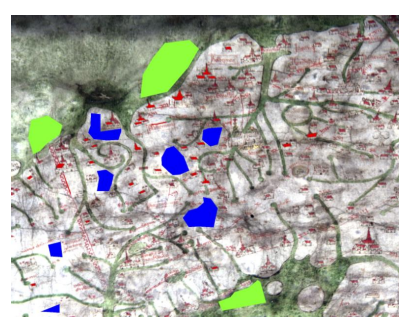

(b)

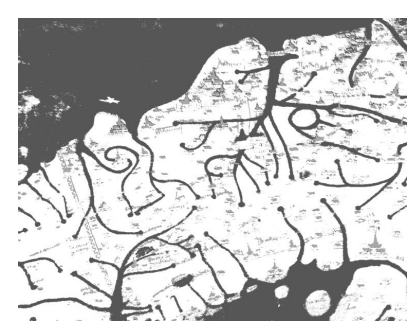

(c)

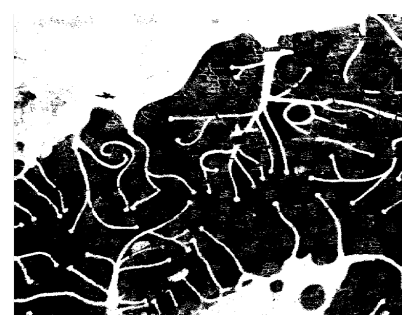

(d)

Figure 8. Example process of extracting green pixels in one sub-chip of the Gough Map. (a) Original 2R(top) chip from the Gough Map; (b) Three regions of interest(ROI) specified in ENVI; (c) Three class Mahalanobis classification result of the $2 \mathrm{R}$ (top) chip; (d) Binary image of $2 \mathrm{R}$ (top) with white pixels representing green pixels of the original $2 \mathrm{R}$ (top) chip. 


\subsubsection{Extract endmembers and estimate material diversity}

The fourth step uses the MaxD and Gram Matrix techniques to extract endmembers and estimate the number of distinct green pigments used for the green pixels of each HSI sub-chip. In Fig. 9(a-l), as described in Messinger et al, (2012), ${ }^{16}$ shown are the plots of the estimated convex hull volume decrease with the increasing number of endmembers (dimensionality) of the data. Since the dimensionality at which the volume function approaches zero indicates the number of distinct materials in the scene, all of the twelve sub-chips show that there are six distinct green pigments in each sub-chip. The similar results is expected since common materials and methodology were likely used across the entire Gough Map.

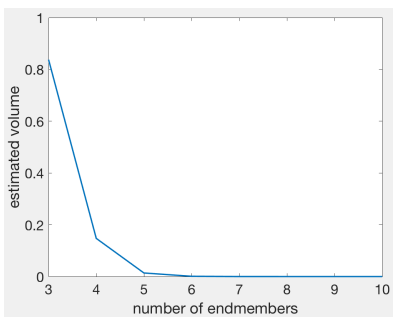

(a)

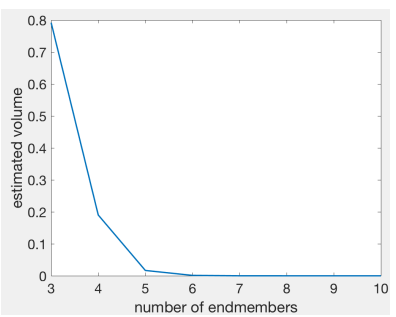

(e)

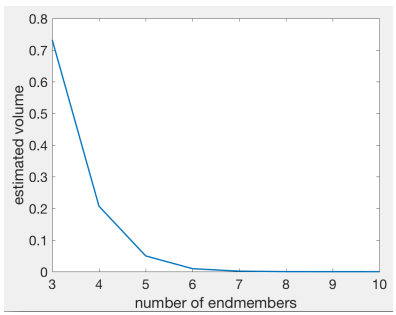

(i)

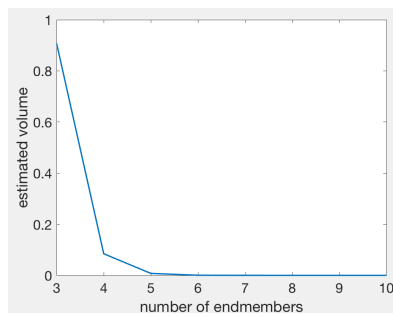

(b)

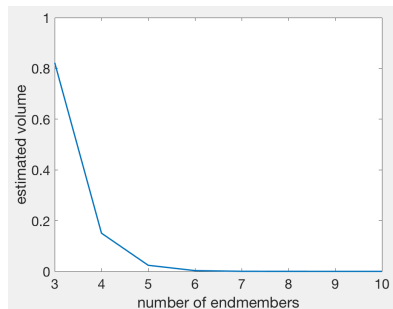

(f)

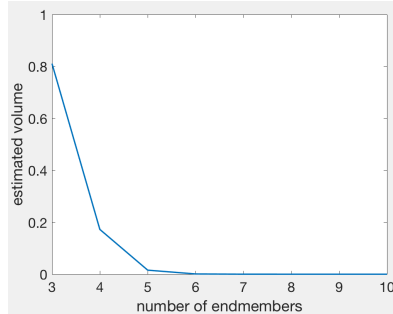

(j)

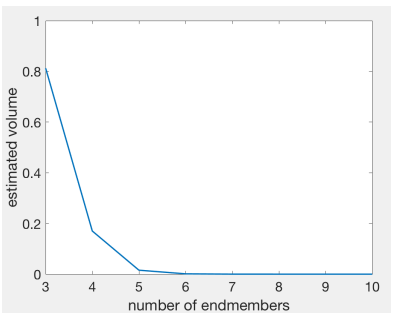

(c)

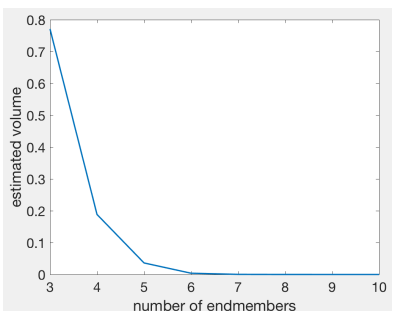

(g)

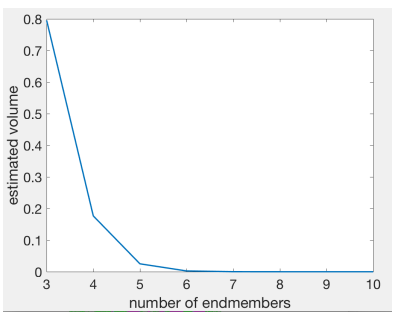

$(\mathrm{k})$

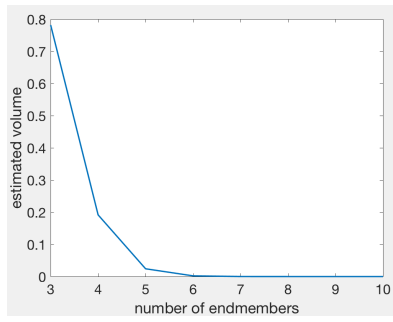

(d)

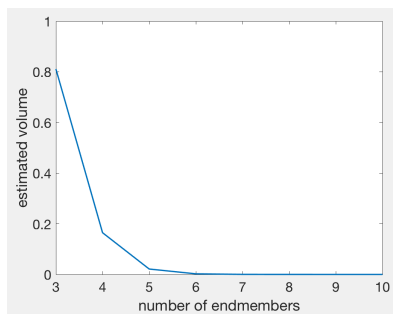

(h)

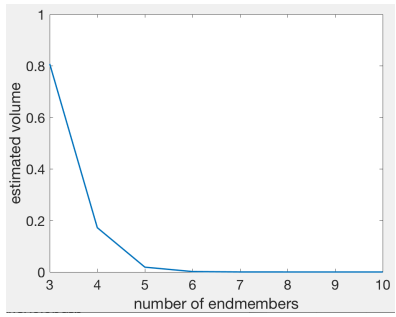

(1)

Figure 9. Material diversity plots for the Gough Map: (a) 3L top (b) 3L bottom (c) 3R top (d) 3R bottom (e) 2L top (f) $2 \mathrm{~L}$ bottom (g) $2 \mathrm{R}$ top (h) $2 \mathrm{R}$ bottom (i) $1 \mathrm{~L}$ top (j) $1 \mathrm{~L}$ bottom (k) $1 \mathrm{R}$ top and (l) $1 \mathrm{R}$ bottom with $\mathrm{x}$ axis: number of endmembers and y axis: estimated convex hull volume.

Given the dimensionality of the green pixels in each sub-chip is six, we output those six endmembers for each sub-chip and plot them in Fig. 10(a-1). Those endmembers are actual spectra of green pixels in each corresponding sub-chip. Notice that the six endmembers for each sub-chip are distinct from each other and represent six unique material per sub-chip. 


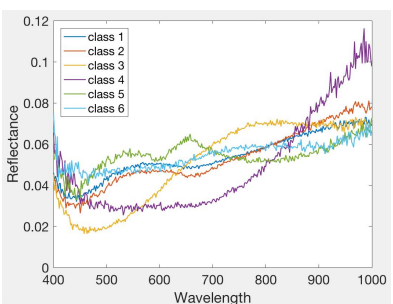

(a)

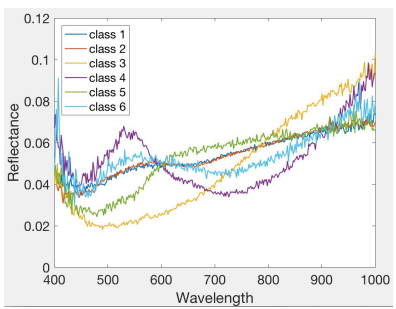

(e)

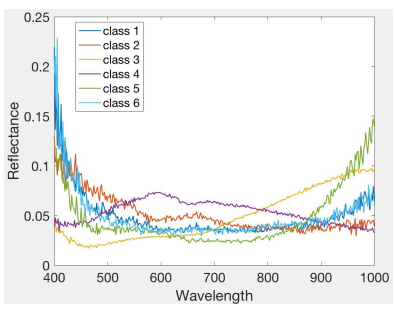

(i)

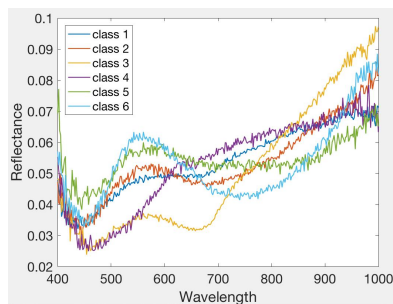

(b)

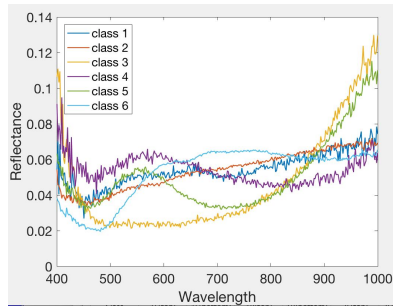

(f)

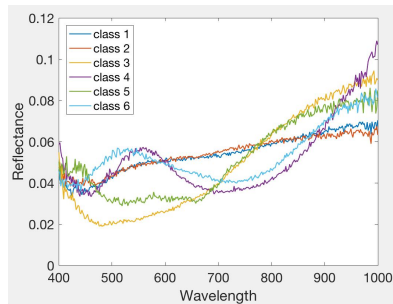

(j)

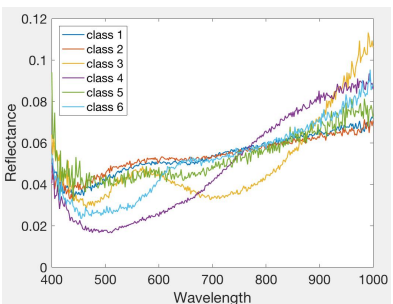

(c)

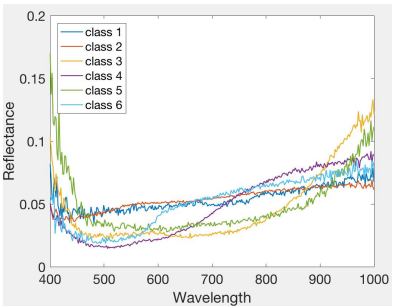

(g)

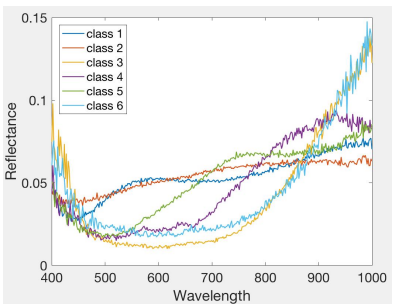

(k)

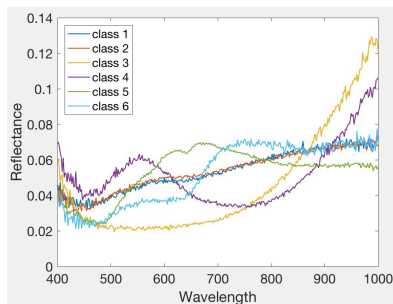

(d)

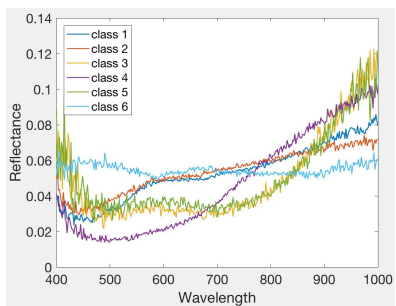

(h)

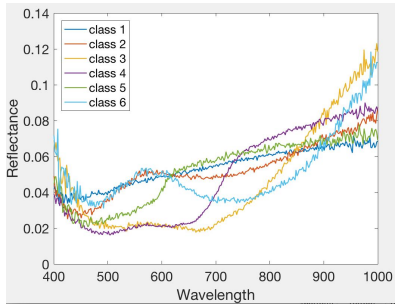

(1)

Figure 10. Endmember plots for the 12 sub-chips of the HSI: (a) 3L top (b) 3L bottom (c) 3R top (d) 3R bottom (e) $2 \mathrm{~L}$ top (f) $2 \mathrm{~L}$ bottom (g) $2 \mathrm{R}$ top (h) $2 \mathrm{R}$ bottom (i) $1 \mathrm{~L}$ top (j) $1 \mathrm{~L}$ bottom (k) $1 \mathrm{R}$ top and (l) $1 \mathrm{R}$ bottom with $\mathrm{x}$ axis as wavelength in $\mathrm{nm}$ and $\mathrm{y}$ axis as reflectance.

\subsubsection{Classify the green pixels per sub-chip}

The fifth step is to classify the green pixels in each sub-chip based on their extracted endmembers. Here, we use spectral angle mapper (SAM) to classify the green pixels within each sub-chip and count the number of pixels in each class. Spectral angle ${ }^{25}$ is defined as

$$
\theta(x, y)=\cos ^{-1}\left(\frac{\sum_{i=1}^{n} x_{i} y_{i}}{\left(\sum_{i=1}^{n} x_{i}^{2}\right)^{\frac{1}{2}} *\left(\sum_{i=1}^{n} y_{i}^{2}\right)^{\frac{1}{2}}}\right)
$$

where $x$ is the spectral vector of a green pixel, $y$ is the spectral vector of an endmember, and $n$ is the number of spectral bands (here $n=334$ ).

For example, the $1 \mathrm{~L}$ (top) chip has six endmembers representing six distinct classes. We calculate the spectral angle between the six endmembers and each green pixel in $1 \mathrm{~L}(\mathrm{top})$, and find out which endmember has the minimum spectral angle (i.e., highest spectral similarity) to this pixel and label it as such. In this way, we classify all the green pixels in each sub-chip and count the number of pixels for each class. The results are shown in Table 1.

From Table 1, note that each sub-chip has some dominant classes, for example, in 1L(top), the third class and fourth class contain most of the green pixels. Although the result of the dimensionality estimation shows that there are 6 different materials in the $1 \mathrm{~L}$ (top), the number of pixels indicates that there are two dominant green materials in this sub-chip. For visual interpretation, we map the results back to the image as described next. 
Table 1. The number of pixels per endmember class for each sub-chip of the Gough Map.

\begin{tabular}{|l|l|l|l|l|l|l|l|l|l|l|l|l|}
\hline Class & $1 \mathrm{~L}($ top) & $1 \mathrm{~L}$ (bot) & $1 \mathrm{R}$ (top) & $1 \mathrm{R}$ (bot) & $2 \mathrm{~L}$ (top) & $2 \mathrm{~L}$ (bot) & $2 \mathrm{R}$ (top) & $2 \mathrm{R}$ (bot) & $3 \mathrm{~L}$ (top) & $3 \mathrm{~L}$ (bot) & $3 \mathrm{R}$ (top) & $3 \mathrm{R}$ (bot) \\
\hline \hline 1 & 2135 & 1242329 & 730530 & 838218 & 330747 & 206521 & 280807 & 750877 & 1740686 & 2372054 & 1850779 & 772161 \\
\hline 2 & 7955 & 191014 & 746860 & 920872 & 2705013 & 2332270 & 1073027 & 571255 & 630102 & 73186 & 360719 & 870361 \\
\hline 3 & 1395183 & 1719 & 15 & 287 & 12203 & 132 & 101 & 1487 & 395 & 12462 & 8046 & 1780 \\
\hline 4 & 350522 & 5606 & 788 & 14620 & 2607 & 334 & 1039 & 12055 & 706 & 2717 & 577 & 4675 \\
\hline 5 & 1000 & 12162 & 12954 & 193369 & 8739 & 18226 & 26 & 7434 & 1376 & 429 & 60335 & 250 \\
\hline 6 & 311 & 100653 & 62 & 3269 & 40685 & 254 & 51641 & 637 & 22969 & 89 & 71781 & 24653 \\
\hline SUM & 1757106 & 1553483 & 1491209 & 1970635 & 3099994 & 2557737 & 1406641 & 1343745 & 2396234 & 2460937 & 2352237 & 1673880 \\
\hline
\end{tabular}

\subsubsection{Visual interpretation: mapping the results back}

The sixth step is to map the classification results per each sub-chip back to the image for visual comparison. The results are shown in Fig. 11(a-l). From the figures, note that most of the sub-chips are mainly composed of two types of green pixels, some sub-chips are dominated by three kinds of green pigments. However, these colors only represent the classification result within each sub-chip. In order to present a distribution pattern of the green pigments across the entire Gough Map, we need to compute global distinct green pigments in the next step.

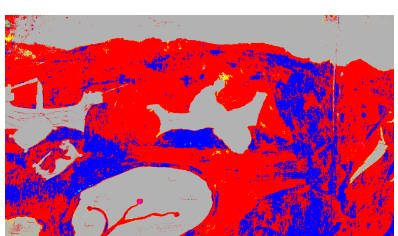

(a)

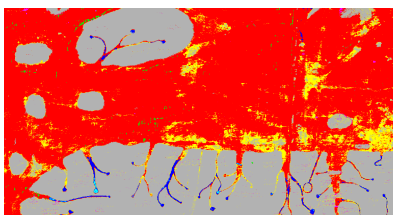

(e)

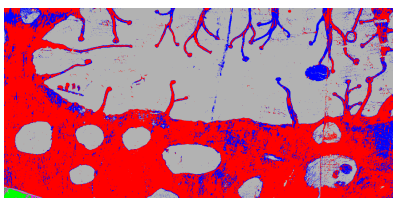

(i)

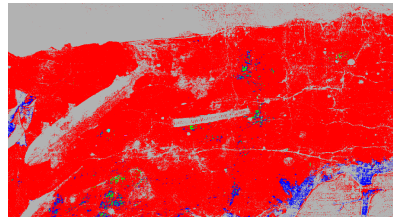

(b)

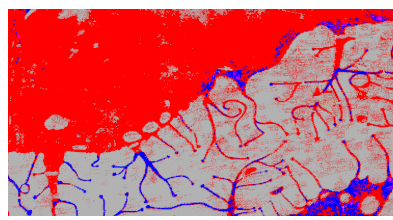

(f)

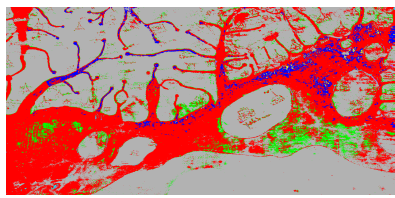

(j)

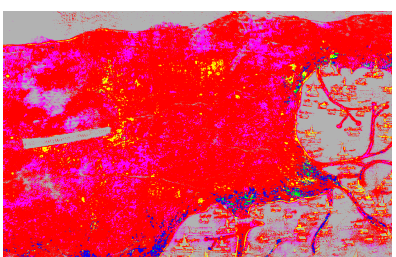

(c)

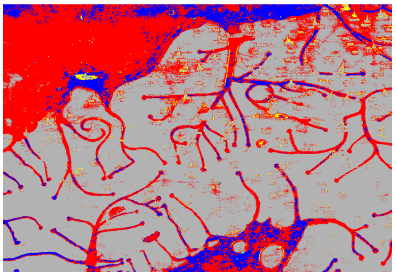

$(\mathrm{g})$

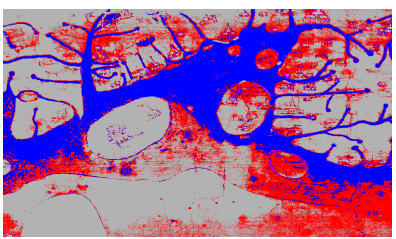

$(\mathrm{k})$

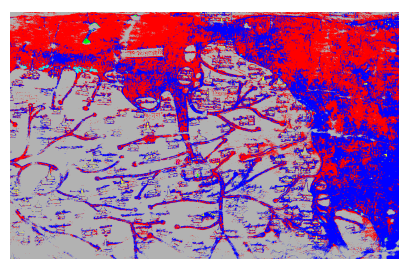

(d)

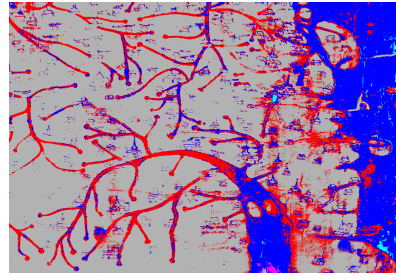

(h)

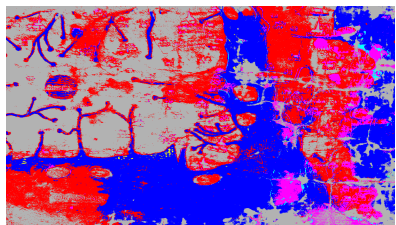

(1)

Figure 11. Green pigments classification patterns of each sub-chip of the Gough Map. (a) 3L top (b) 3L bottom (c) 3R top (d) $3 \mathrm{R}$ bottom (e) $2 \mathrm{~L}$ top (f) $2 \mathrm{~L}$ bottom (g) $2 \mathrm{R}$ top (h) $2 \mathrm{R}$ bottom (i) $1 \mathrm{~L}$ top (j) $1 \mathrm{~L}$ bottom (k) $1 \mathrm{R}$ top and (l) $1 \mathrm{R}$ bottom. 


\subsubsection{Compute global distinct green pigments for the entire Gough Map}

The last step is to identify distinct green pigments in the entire Gough Map. First, we need to use the MaxD and Gram Matrix approach to estimate the number of distinct green pigments from the 72 primary endmembers depicted in 3.2.4. In Fig. 12, shown is the plot of estimated volume with increasing number of endmembers of the 72 primary classes (endmembers). It depicts that there should be 6 distinct green pigments used to represent the entire Gough Map.

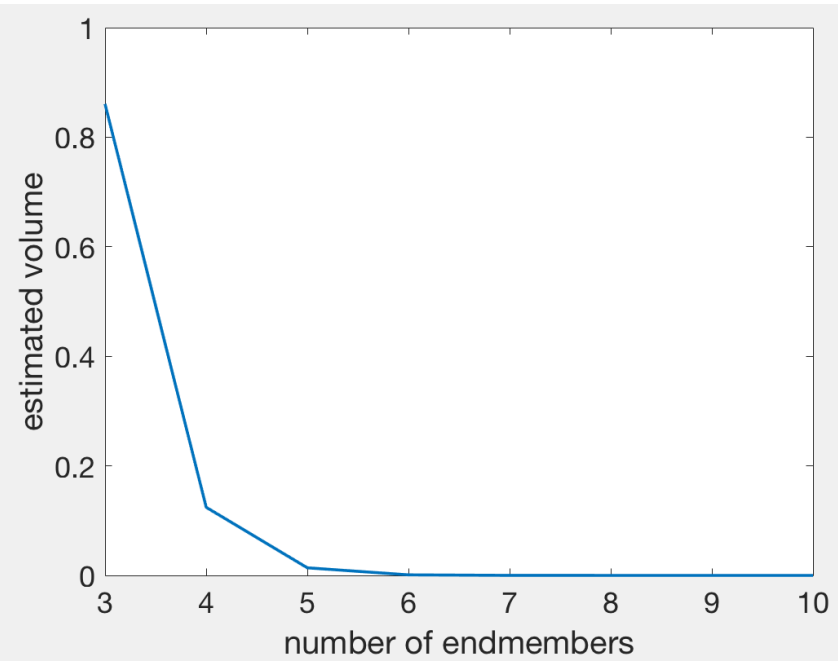

Figure 12. Complexity plot for the 72 classes with $\mathrm{x}$ axis: number of endmembers and y axis: estimated convex hull volume.

Since the dimensionality of the green pixels in the entire Gough Map is six, our second step is to output those six endmembers for the green pixels. Different from section 3.2.3, we do not simply output six endmembers from the 72 primary classes because we are only interested in dominant classes. Here, the dominant classes are such that have more than 40,000 pixels associated to their class in that sub-chip. Consequently, we only consider the following within-chip classes shown in the gray-labeled cells in Table 2. In total, we consider 30 dominant classes from the individual sub-chips.

Table 2. The number of pixels per class for the Gough Map with dominant classes labeled in gray.

\begin{tabular}{|c|c|c|c|c|c|c|c|c|c|c|c|c|}
\hline Class & 1L(top) & 1L(bot) & 1R(top) & 1R(bot) & $2 \mathrm{~L}$ (top) & $2 \mathrm{~L}$ (bot) & $2 \mathrm{R}$ (top) & $2 \mathrm{R}$ (bot) & $3 \mathrm{~L}$ (top) & $3 \mathrm{~L}$ (bot) & $3 \mathrm{R}$ (top) & $3 \mathrm{R}$ (bot) \\
\hline 1 & 2135 & 1242329 & 730530 & 838218 & 330747 & 206521 & 280807 & 750877 & 1740686 & 2372054 & 1850779 & 772161 \\
\hline 2 & 7955 & 191014 & 746860 & 920872 & 2705013 & 2332270 & 1073027 & 571255 & 630102 & 73186 & 360719 & 870361 \\
\hline 3 & 1395183 & 1719 & 15 & 287 & 12203 & 132 & 101 & 1487 & 395 & 12462 & 8046 & 1780 \\
\hline 4 & 350522 & 5606 & 788 & 14620 & 2607 & 334 & 1039 & 12055 & 706 & 2717 & 577 & 4675 \\
\hline 5 & 1000 & 12162 & 12954 & 193369 & 8739 & 18226 & 26 & 7434 & 1376 & 429 & 60335 & 250 \\
\hline 6 & 311 & 100653 & 62 & 3269 & 40685 & 254 & 51641 & 637 & 22969 & 89 & 71781 & 24653 \\
\hline SUM & 1757106 & 1553483 & 1491209 & 1970635 & 3099994 & 2557737 & 1406641 & 1343745 & 2396234 & 2460937 & 2352237 & 1673880 \\
\hline
\end{tabular}

Now we need to choose more dominant classes from the 30 classes. We use the spectral angle to re-classify the green pixels in each sub-chip of the Gough Map based on the 30 dominant classes. Due to the similarities between some classes, results show that there are 18 dominant green pigments in the 30 classes, which are $1 \mathrm{~L}(\mathrm{bottom})$ :class 1,$2 ; 1 \mathrm{R}$ (top):class $2 ; 1 \mathrm{R}$ (bottom):class 1,2; 2L(top):class $2 ; 2 \mathrm{~L}$ (bottom):class $2 ; 2 \mathrm{R}$ (top):class 2; 2R(bottom):class 1,2; 3L(top):class 1,2; 3L(bottom):class 1,2; 3R(top):class 1; 3R(top):class 6; 3R(bottom):class 1,2. Since Fig. 12 indicates six distinct green pigments in the entire Gough Map, we can use K-means classification to classify the spectra of the 18 dominant classes into six clusters. The classification results for 18 classes are shown in Fig. 13. 


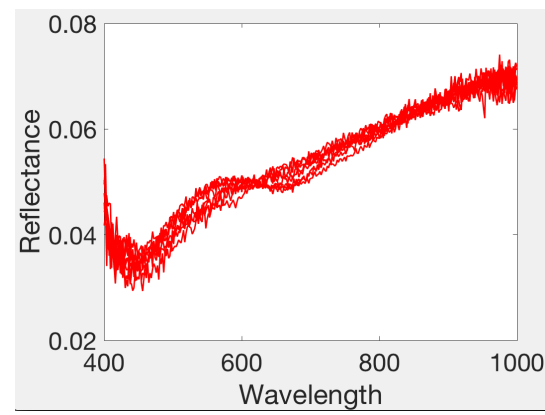

(a)

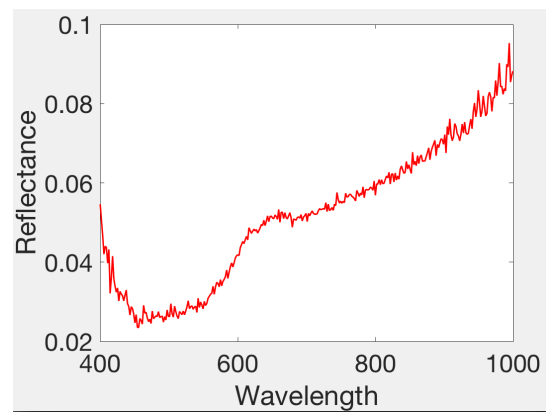

(d)

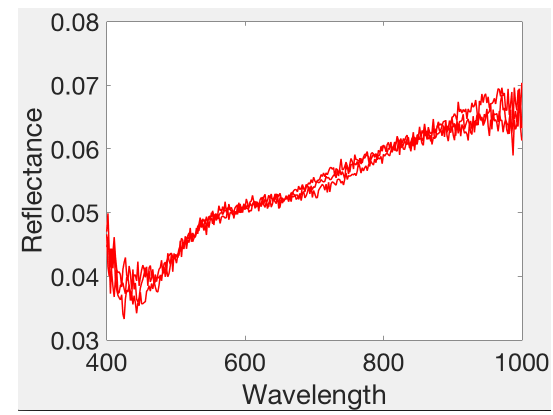

(b)

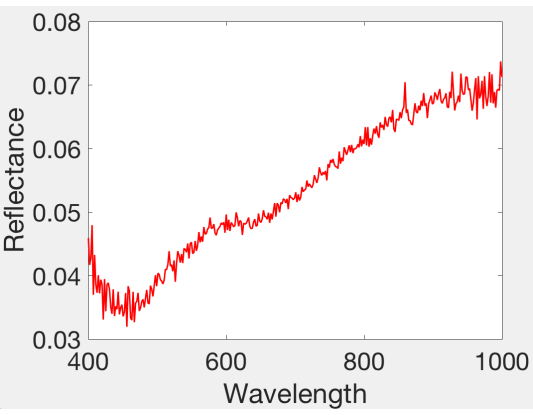

(e)

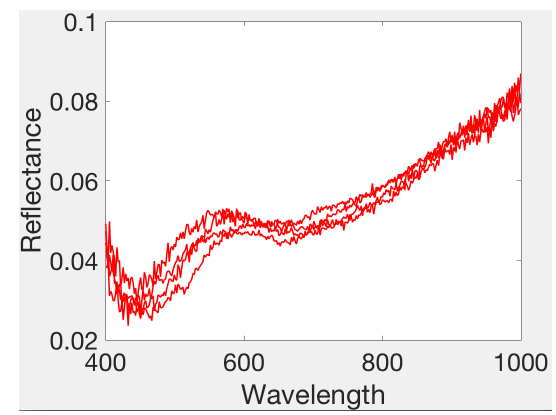

(c)

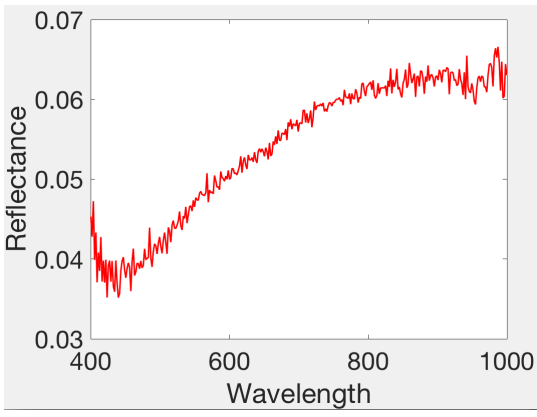

(f)

Figure 13. K-means classification results of 18 dominant classes of the green pixels.

After computing the average spectra within each class, we have six distinct global endmembers for the green pixels in the entire Gough Map. Fig. 14 presents the results of the spectra of the six distinct green pigments.

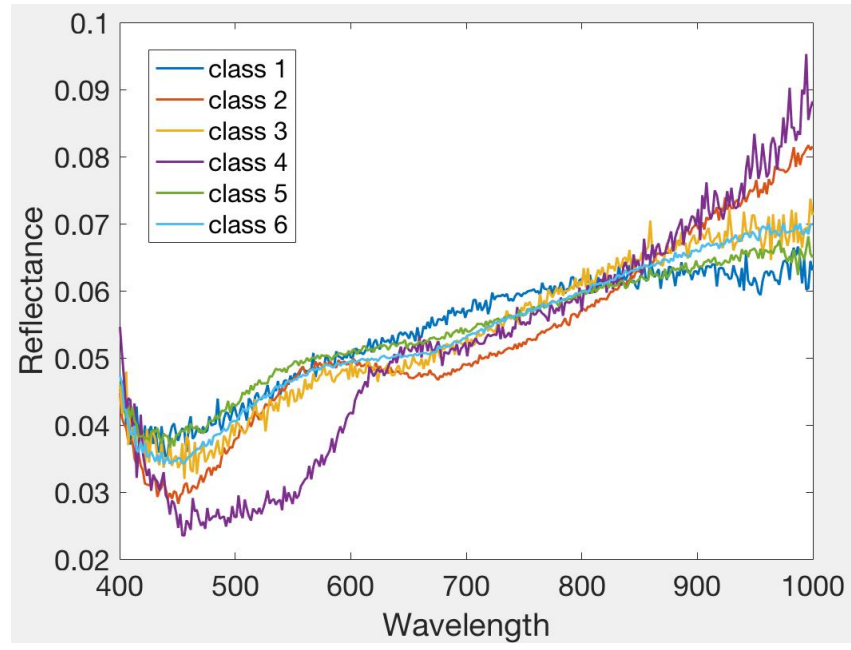

Figure 14. Spectra of six dominant classes of the green pixels in the Gough Map.

Now we use spectral angle mapper (SAM) to re-classify the green pixels based on the six global dominant classes per sub-chip and map the results back to the image for visual comparison. The results of pixels count for each class is shown in Table 3. Notice that the number of pixels per each class distributes evenly this time, which indicates that the six global dominant classes separate the green pixels pretty well. 
Table 3. The number of green pixels per global dominant class for each HSI sub-chip of the Gough Map.

\begin{tabular}{|c|c|c|c|c|c|c|c|c|c|c|c|c|}
\hline Class & $1 \mathrm{~L}$ (top) & $1 \mathrm{~L}$ (bot) & $1 \mathrm{R}$ (top) & 1R(bot) & $2 \mathrm{~L}$ (top) & 2L(bot) & $2 \mathrm{R}$ (top) & 2R(bot) & $3 \mathrm{~L}$ (top) & 3L(bot) & $3 \mathrm{R}$ (top) & $3 \mathrm{R}$ (bot) \\
\hline 1 & 402122 & 398876 & 285836 & 54103 & 1566824 & 1021006 & 399722 & 56901 & 1137308 & 1243075 & 943069 & 192348 \\
\hline 2 & 18204 & 14873 & 18750 & 265470 & 4937 & 17865 & 29915 & 255188 & 5851 & 615 & 17194 & 87508 \\
\hline 3 & 93676 & 336903 & 310733 & 48743 & 94042 & 328031 & 348084 & 90613 & 102657 & 40986 & 188568 & 115954 \\
\hline 4 & 105599 & 258340 & 304363 & 73269 & 209673 & 274524 & 145284 & 4251 & 297605 & 556923 & 484417 & 109675 \\
\hline 5 & 544319 & 119706 & 117904 & 744281 & 803063 & 409086 & 189785 & 522083 & 504285 & 232222 & 244015 & 452743 \\
\hline 6 & 593186 & 424785 & 453623 & 784769 & 421455 & 507225 & 293851 & 414709 & 348528 & 387116 & 474974 & 715652 \\
\hline SUM & 1757106 & 1553483 & 1491209 & 1970635 & 3099994 & 2557737 & 1406641 & 1343745 & 2396234 & 2460937 & 2352237 & 1673880 \\
\hline
\end{tabular}

For visual comparison, we combine twelve sub-chips into one image and the final classification result is shown in Fig. 15. Class 1 is interpreted in red; class 2: blue; class 3: green; class 4: yellow; class 5: magenta and class 6: cyan. From Fig. 15, notice that most of the ocean area is in red, magenta, yellow and cyan, most of the coastal and waterways are in green and some faded area on the right side of the Gough Map are in blue and magenta. If we compare this result with Fig. 1 (the original RGB image of the Gough Map), notice that the ocean area is painted with a mix of light and dark green ink, the coastal and waterways are drawn with another kind of dark green ink and the faded area on the right looks even darker. Out final classification result matches what we see in the original RGB image.

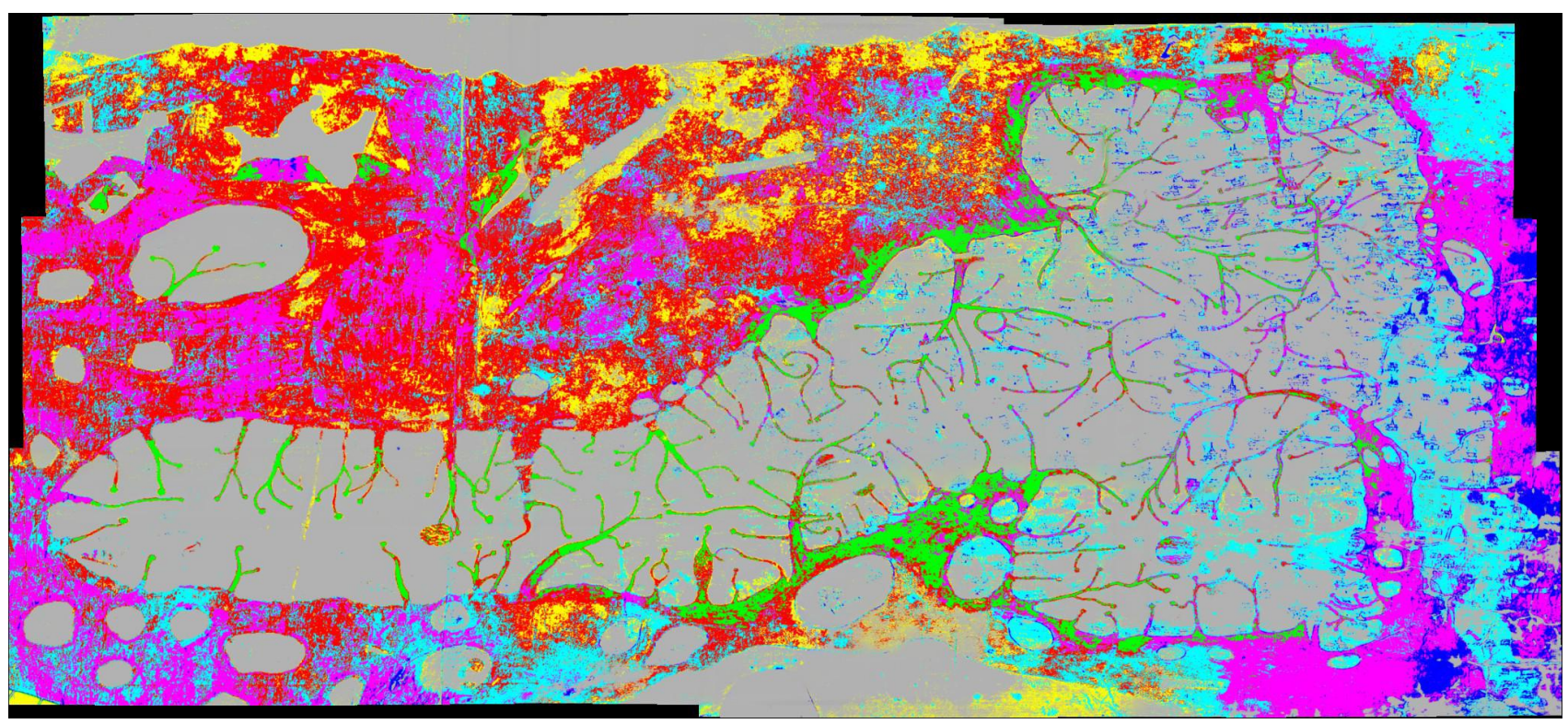

Figure 15. Classification result of HSI of the entire Gough Map (combination of 12 sub-chips).

\section{SUMMARY}

This research considers a novel application for hyperspectral imagery: analysis of pigment diversity in a medieval map. We have developed a pigment analysis tool for a set of hyperspectral images of the Gough Map, one of the earliest surviving maps of Britain in geographically recognizable form. The map was likely created in the 15th century and revised over decades. The methodology and motivation for its creation are largely unknown. The goal of this work is to estimate the material diversity of the green pigments so as to contribute to the codicological studies of the Gough Map, i.e., understanding the techniques, materials used in its creation and potentially its creation and revision timeline. Green (visually) pixels are obtained from each of the 12 sub-chips of 
the entire map. The Gram matrix and MaxD techniques are used to estimate the dimensionality and compute the endmembers of each sub-chip. Dominant classes are then picked out and combined into six global endmembers (distinct materials). All green pigments in the map are classified into one of these six global classes and their spatial distribution was assessed by mapping them back into the original image so that global patterns are easily identified. The conclusion of this research is that there are six unique green pigments present in the Gough Map. In addition, we can confidently say that the primary differences in the green pigments are between the coastal area, waterways and the ocean. Apparently, there is some spatial relationship to the distribution of some of the green pigment classes that could be related to the timeline of the construction and revision of the Gough Map. This methodology can not only be applied to the green pigments to assess their within-material diversity, but also be generalized to a novel pigment analysis tool for historical geographers and cartographic historians to analyze the material diversity of the hyperspectral images of cultural heritage artifacts and consequently, contribute to the codicological study of the cultural heritage.

\section{ACKNOWLEDGMENTS}

The authors wish to acknowledge Dr. Catherine Delano-Smith and Mr. Damien Bove for their insightful comments into the history, significance, and questions about the Gough map. We also wish to acknowledge Mr. Nick Millea at the Bodleian Library, Oxford University for his assistance with this project.

\section{REFERENCES}

[1] Solopova, E., "The making and re-making of the gough map of britain:manuscript evidence and historical context," Imago Mundi 64:2, 155-168 (2012).

[2] Lilley, K. D., Lloyd, C. D., and Campbell, B. M. S., "Mapping the realm: A new look at the gough map of britain cartographic veracity in medieval mapping: Analyzing geographical variation in the gough map of great britain," Imago Mundi 61:1, 1-28 (2009).

[3] Delano-Smith, C., Barber, P., Bove, D., Clarkson, C., Harvey, P., Millea, N., an W. Shannon, N. S., Whittick, C., and Willoughby, J., "New light on the medieval gough map of britain," Imago Mundi 69:1, $1-36(2016)$.

[4] MILLEA, N., [The Gough map: the earliest road map of Great Britain.], The Bodleian Library, Oxford (2007).

[5] Easton, R. L., Barry, W. A. C., and Knox, K. T., "Ten years of lessons from imaging of the archimedes palimpsest," Commentationes Humanarum Litterarum. 129, 5-34 (2011).

[6] Easton, R. L. and Noel, W., "Infinite possibilities: Ten years of study of the archimedes palimpsest," Proceedings of the American Philosophical Society 154, 50-76 (2010).

[7] Barry, W. A. C., Boydston, K., and Easton, R. L., "Some properties of textual heritage materials of importance in spectral imaging projects," Commentationes Humanarum Litterarum. 129, 35-50 (2011).

[8] Barry, W. A. C., Boydston, K., and Easton, R. L., "Some properties of textual heritage materials of importance in spectral 27 imaging projects," Proceedings of Eikonopoiia, Eikonopoiia, Digital Imaging of Ancient Textual Heritage: Technological Challenges and Solutions, Eikonopoiia 1, 27-38 (2010).

[9] Knox, K. T., Easton, R. L., Barry, W. A. C., and Boydston, K., "Recovery of handwritten text from the diaries and papers of david livingstone," Proceedings of the SPIE, Electronic Imaging 7689, 786909-786909 (2011).

[10] Easton, R. L. and Kelbe, D., "Statistical processing of spectral imagery to recover writings from erased or damaged manuscripts," Manuscript Cultures. 7, 35-46 (2014).

[11] Easton, R. L. and Noel, W., "Multispectral imaging of the archimedes palimpsest," Gazette du Livre Medieval. 45, 39-49 (2004).

[12] Easton, R. L., Sacca, K., Heyworth, G., Boydston, K., Duzer, C. V., and Phelps, M., "Rediscovering text in the yale martellus map, spectral imaging and the new cartography," Proceedings of the 7th IEEE International Workshop on Information Forensics and Security, 1-6 (2015).

[13] Zhao, Y., Image segmentation and pigment mapping of cultural heritage based on spectral imaging, $\mathrm{PhD}$ thesis, Rochester Institute of Technology, Rochester, NY (2008). 
[14] Comelli, D., Valentini, G., and Cubeddu, R., "Multi-spectral fluorescence imaging for cultural heritage," in [Optics for Arts, Architecture, and Archaeology], Fotakis, C., Pezzati, L., and Salimbeni, R., eds., Proceedings of the SPIE 6618, 66180M1-66180M9 (2007). [doi:10.1117/12.726077].

[15] Camastra, F., "Data dimensionality estimation methods: A survey," Pattern Recognition 36, 2945-2954 (2003).

[16] Messinger, D. W., Ziemann, A., Basener, B., and Schlamm, A., "Metrics of spectral image complexity with application to large area search," Optical Engineering 51(3), 036201-1-036201-9 (2012).

[17] Canham, K., Schlamm, A., Ziemann, A., Basener, B., and Messinger, D. W., "Spatially adaptive hyperspectral endmember selection and spectral unmixing," IEE Trans. on Geoscience and Remote Sensing 49(11), 4248-4262 (2011).

[18] Kirby, M., [Geometric Data Analysis: An Empirical Approach to Dimensionality Reduction and the Study of Patterns], John Wiley \& Sons, Inc., New York, NY, USA (2000).

[19] Jolliffe, I., [Principal Component Analysis.], Springer-Verlag New York, New York (2002).

[20] Ziemann, A. K., Messinger, D. W., and Basener, W. F., "Iterative convex hull volume estimation in hyperspectral imagery for change detection," Proceedings of the SPIE 7695, 76951I-76951I-9 (2010).

[21] Yao, F. and Qian, Y., "Band selection based gaussian processes for hyperspectral remote sensing images classification," 2009 16th IEEE International Conference on Image Processing (ICIP) , 2845-2848 (Nov 2009).

[22] Pettis, K. W., Bailey, T. A., Jain, A. K., and Dubes, R. C., "An intrinsic dimensionality estimator from near-neighbor information," IEEE Transactions on Pattern Analysis and Machine Intelligence PAMI-1, 25-37 (Jan 1979).

[23] Schlamm, A., Messinger, D., and Basener, B., "Geometric estimation of the inherent dimensionality of single and multi-material clusters in hyperspectral imagery," Journal of Applied Remote Sensing 3, 033527033527-16 (2009).

[24] Schott, J. R., Lee, K., Raqueno, R., Hoffmann, G., and Healey, G., "A subpixel target detection technique based on the invariance approach," Proceedings of the AVIRIS Workshop, NASA, JPL, Pasadena, CA, (Feb. 2003).

[25] Kruse, F. A., Lefkoff, A. B., Boardman, J. B., Heidebrecht, K. B., Shapiro, A. T., Barloon, P. J., and Goetz, A. F. H., "The spectral image processing system (sips) - interactive visualization and analysis of imaging spectrometer data," Remote Sensing of Environment. 44, 145-163 (1993). 